\title{
HIGH RESOLUTION OBSERVATIONS OF CO IN PNe
}

\author{
K.M. SHIBATA and S. DEGUCHI \\ Nobeyama Radio Observatory, National Astronomical Observatory, Japan \\ T. KASUGA \\ Department of Instrument and Control Engineering, Hosei University, Japan \\ S. TAMURA \\ Astronomical Institute, Tohoku University, Japan \\ N. HIRANO \\ Laboratory of Astronomy and Geophysics, Hitotsubashi University, Japan \\ and \\ O. KAMEYA \\ Mizusawa Astrogeodynamics Observatory, Japan
}

\begin{abstract}
We have made aperture synthesis observations of ${ }^{12} \mathrm{CO}(\mathrm{J}=1-0)$ emission IRAS 21282+5050, CRL 618 and M 1-7 using the Nobeyama Millimeter Array (NMA). We observed with 3 or 2 configurations and obtained an angular resolution of 3" 2 x 3.1, 3". 6 x 3" 5 and 4". 3 x 3" 8 .

In CRL 618, the CO emission has a size of about $15 " \times 12$ " and concentrates to the center of the continuum emission. CO emission extends from the NW to the $\mathrm{SE}$ at the lower contours. At the higher contours it elongates to the EW and has a weak extension to the $S$ at the western part of the emission. In the velocity channel maps, the emissions have only one peak and change complexly their shape at each velocity. No systematic trend with velocity can be seen in these maps. As a first approximation the CO gas in CRL 618 has a spherical structure in its base. It is considered that the ionized cavity in the molecular gas cannot be resolved because of its small size. The total flux density of the continuum emission at $115 \mathrm{GHz}$ is $1.48 \mathrm{Jy}$. We may consider that all the $1.48 \mathrm{Jy}$ come from a $\mathrm{f}-\mathrm{f}$ emission of the ionized gas. Our value is consistent with the cylindrical isothermal ionized gas model with a power-law density distribution (Martín-Pintado et al. 1988).

The integrated CO map in M 1-7 shows that the CO emission elongates toward a p.a. $=60^{\circ}$ (perpendicular to the optical major axis) and has a size of about $10 "$ $x$ 20". Two emission peaks are shown in each side of the nebular center. Pos.-vel. diagrams clearly show the toroidal structure around the minor axis of the optical image. The CO structure is very similar to that of NGC 2346 (Bachiller et al. 1989). Obviously, M 1-7 is in an earlier evolutionary stage than NGC 2346 and its ionized gas will develop faster toward the pole of the $\mathrm{CO}$ toroid than toward the equatorial direction and then become a bipolar nebula like NGC 2346.

For IRAS 21282, we conclude that CO gas forms an expanding toroid whose axis lies along the EW direction and is normal to the line of sight. Both from the ratio of the size of $\mathrm{CO}$ gas to that of ionized gas and from the intensity ratio of [OIII] to $\mathrm{H} \beta$, we conclude that IRAS $21282+5050$ is in an earlier evolutionary stage than NGC 7027 and 2346. Detailed discussions are given in Shibata et al. (1989).

\section{References}

Bachiller et al., 1989, Astron. Ap., 210, 366.

Martín-Pintado et al., 1988, Astron. Ap., 197, L15.

Shibata et al., Ap. J. (Letters), 345, L55. 